\title{
Protocol
}

\section{Using WebGBrowse to Visualize Genome Annotation on GBrowse}

\author{
Ram Podicheti and Qunfeng Dong ${ }^{1}$ \\ Center for Genomics and Bioinformatics, Indiana University, Bloomington, IN 47405, USA
}

\section{INTRODUCTION}

Although GBrowse is popular for visualizing genomic features along a reference sequence, its installation and configuration are difficult for many biologists. WebGBrowse is a web server that takes a usersupplied annotation file, guides users to configure the display of each genomic feature, and allows users to visualize the genome annotation with integrated GBrowse software. This protocol guides the user through each step of using WebGBrowse.

\section{RELATED INFORMATION}

An exhaustive and illustrated tutorial is available on the WebGBrowse website at http:// webgbrowse.cgb.indiana.edu/tutorial.html. A GBrowse user introductory tutorial is available on OpenHelix at http://www.openhelix.com/gbrowse. The format specification for Generic Feature Format version 3 (GFF3) is available at http://www.sequenceontology.org/gff3.shtml.

\section{MATERIALS}

CAUTIONS AND RECIPES: Please see Appendices for appropriate handling of materials marked with $<!>$, and recipes for reagents marked with $<R>$.

Equipment

Genome annotation file in GFF3 format

Internet-connected computer with web browser

\section{METHOD}

The procedure below describes how to display a typical genome annotation on GBrowse (Stein et al. 2002) at the WebGBrowse (Podicheti et al. 2009) server. Some nomenclature is defined in Table 1.

\section{Uploading the Genome Annotation Data Set}

1. Use any modern web browser (e.g., Firefox) to open the URL http://webgbrowse.cgb.indiana.edu. You are now at the WebGBrowse Input Form (Fig. 1).

2. Click the link "[Sample GFF3 File]" to download the sample data set, volvox.gff3 (Fig. 2).

This data set presents typical feature types that can be configured to illustrate the default generic display, protein-coding genes, quantitative data display, and so on. We suggest trying the sample data set first before uploading your own GFF3 data set.

${ }^{1}$ Corresponding author (qunfengd@gmail.com). 
Table 1. Genome browser nomenclature

\begin{tabular}{ll}
\hline Term & Definition \\
\hline Feature & $\begin{array}{l}\text { Sequence characteristic (e.g., gene) that can be physically mapped to reference genome. } \\
\text { Track }\end{array}$ \\
& $\begin{array}{l}\text { Horizontal display of instances of a particular feature type. Tracks are located under the refer- } \\
\text { ence genomic sequence. For example, two tracks are used to display gene and mRNA features } \\
\text { in Figure } 6 .\end{array}$ \\
Glyph & $\begin{array}{l}\text { Shape of the feature diagram. A WebGBrowse-supported glyph library is available at } \\
\text { http://webgbrowse.cgb.indiana.edu/glyphdoc.html. }\end{array}$ \\
Configuration & GBrowse display settings (e.g., feature tracks, glyph, font, and color).
\end{tabular}

3. Click the button "Browse..." in the "GFF3 File" section and upload the sample file (volvox.gff3).

4. (Optional) In the text input field under "Email address," enter your e-mail address.

This allows you to perform the configuration in multiple sessions, have the configuration results sent to your e-mail address, and keep track of all your previous submissions.

5. Click the button "Submit" to send your data to WebGBrowse. WebGBrowse will validate your input and redirect you to the Configuration Panel.

For this example, leave the input field blank for "Configuration File to be used as a template." Use of this feature is described in Step 18.

\section{Working in the Configuration Panel}

The Configuration Panel allows you to add, edit, or delete feature tracks for the GBrowse display of your data set.

6. Provide a short description for your data set in the "Description" field.

7. From the section "Add New Track" (Fig. 3), select a feature from the Feature menu. The Feature menu lists all the unique features derived from the data set that can be configured into individual GBrowse tracks.

8. For each selected feature, choose its shape by selecting from the pull-down Glyph menu. A glyph library with a sample image and short description for each selected glyph is also displayed.

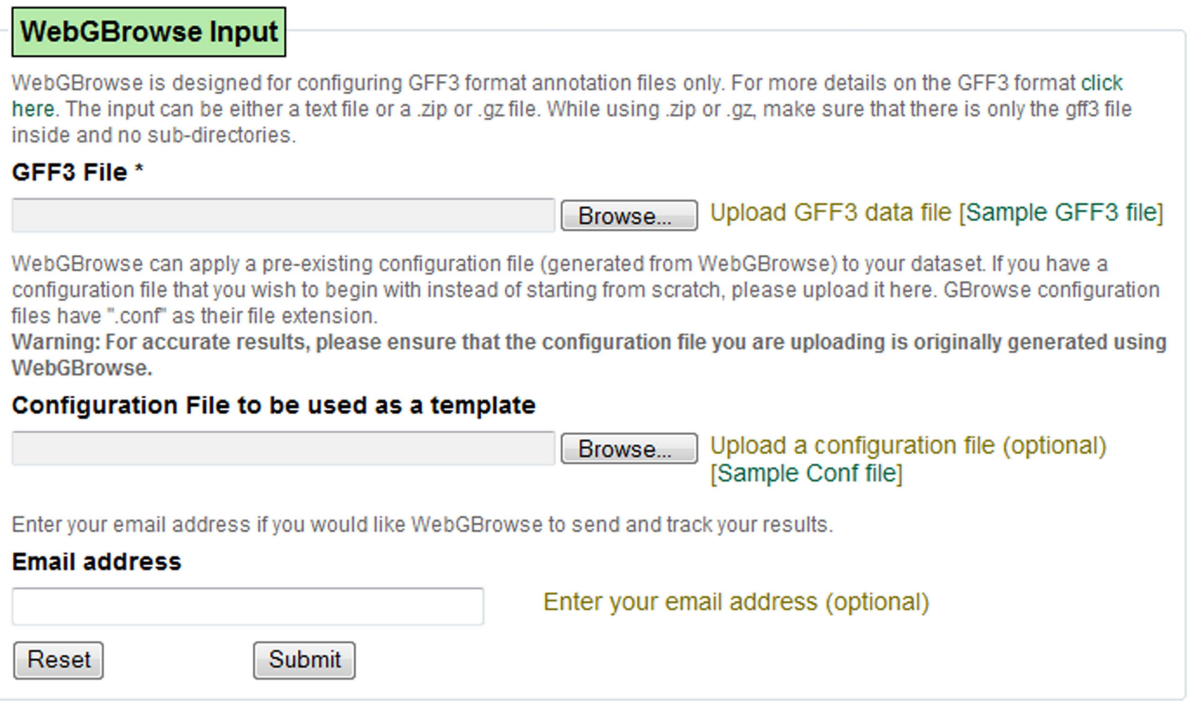

FIGURE 1. The WebGBrowse Input Form allows users to provide the genome annotation data sets (GFF3 format), configuration templates, and their e-mail address. 
\#\#ff-version 3

* file: volvox.gff3 derived from GBrowse Administration Tutorial by Lincoln Stein, 2008

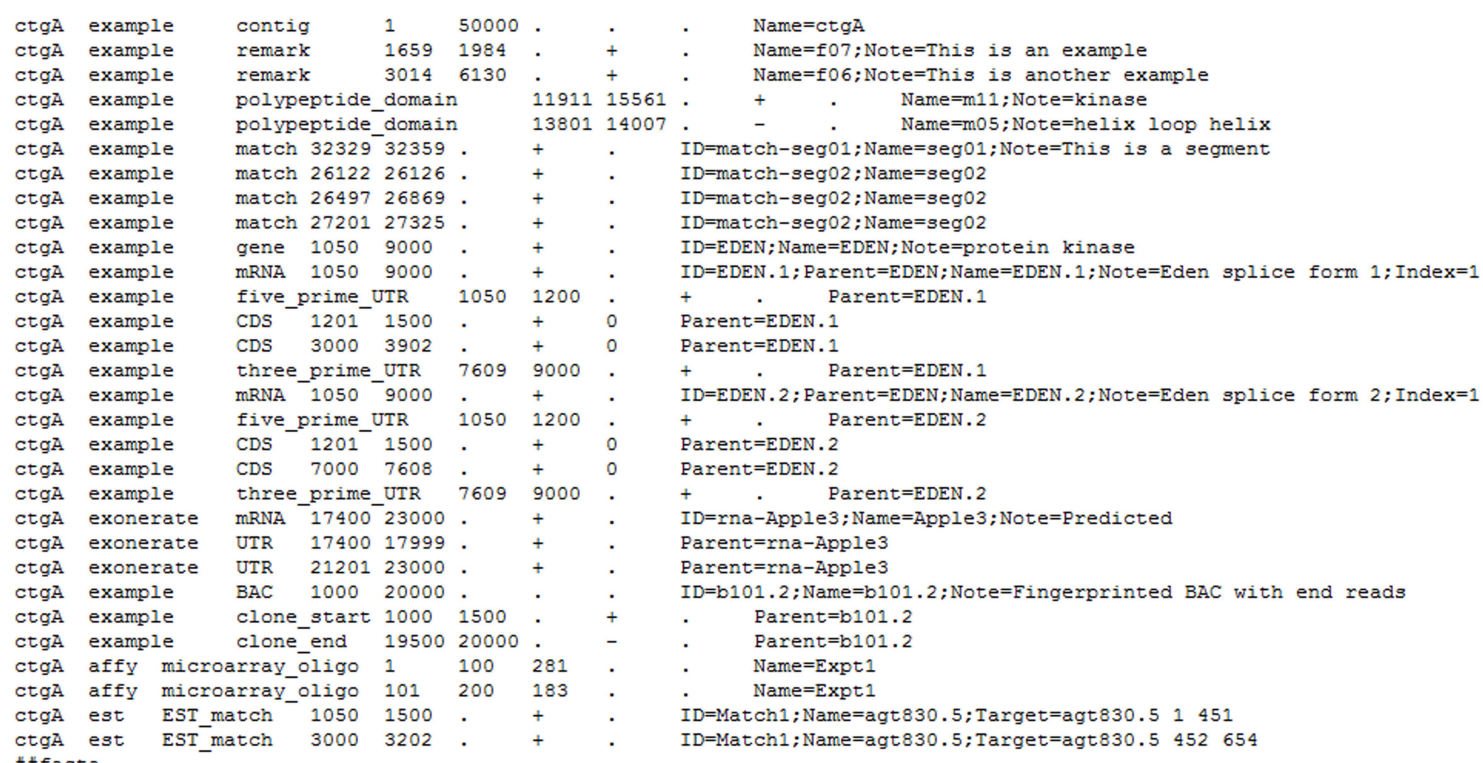

\#fasta

$>\operatorname{ctgA}$

cattgttgcggagttgaacaacggcattaggaacacttccgtctctcactttatacgat

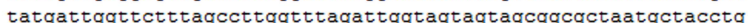

aattgagaactcgagcgggggctaggcaaattctgattcagcctgacttctcttggaacc

ctgcccataaatcaaagggttagtgcggccaaacgttggacaacggtattagaagacca

acctgaccaccaaaccgtcaattaaccggtatcttctcggaaacggcggttctctcctag

atagcgatctgtggtctcaccatgcaatttaaacaggtgagtaaagattgctacaatac

gagactagctgtcaccagatgctgttcatctgttggctcettggtcgctccgttgtaccc

FIGURE 2. The sample GFF3 file was modified from the GFF3 example provided in the GBrowse installation package. This figure displays partial contents of the sample GFF3 file due to space limitations. GFF3 is a standard format for representing genomic annotation, which consists of nine tab-delimited columns that specify each sequence feature (e.g., gene, mRNA, coding sequence [CDS], untranslated region [UTR], etc., in column 3) and its corresponding genomic coordinates (start and end positions in column 4 and column 5, respectively). The actual DNA sequence can also be provided in a GFF3 file (e.g., the sequence for the genomic contig ctgA). For complete GFF3 specifications, see http://www.sequenceontology.org/gff3.shtml.

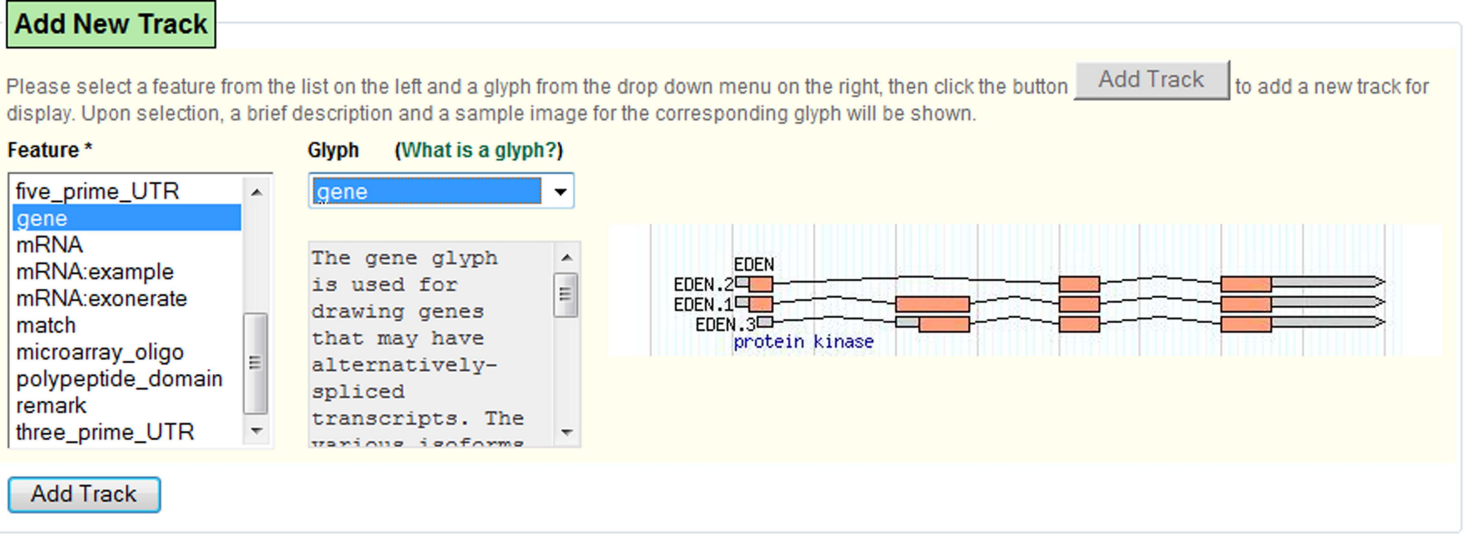

FIGURE 3. Genomic features extracted from the GFF3 file are displayed in the Features box of the Configuration Panel. For each feature, users can select a display style from the Glyph drop-down menu. Each glyph is illustrated with a brief description and an example image. Each Feature-Glyph pair defines a track in the GBrowse display. 
9. Click the button "Add Track." This will open the floating Glyph Parameters Form (Fig. 4), where the parameters for the selected glyph are displayed.

The presented configurable parameter set is specific to the type of glyph chosen. Each parameter field has a brief description explaining the purpose of the parameter. If you have provided your e-mail address in Step 4, you will see a "Save Progress" button at the top right corner of the configuration panel that lets you save your progress and complete the configuration in a later session. You can save your progress at any stage by clicking on the "Save Progress" button and WebGBrowse will e-mail you a link, which can be later followed to resume your work.

10. Use the Glyph Parameters Form to change any of the default parameter values (e.g., the color of the displayed track), if desired. More parameters can be viewed by clicking the link "Advanced Section."

11. Once you are done setting the parameter values, click the button "Save and Continue" to go back to the Configuration Panel.

The configured tracks and their corresponding configuration settings will be listed under the section "Tracks Added" in the Configuration Panel (Fig. 5).

12. Repeat Steps 7-11 to add all the desired tracks.

13. To edit any existing track configuration settings, select the track in the section "Tracks Added" and click the button "Edit Track."

14. To delete a track, click the button "Delete Track."

15. After adding and configuring all tracks, click the button "Display in GBrowse" to visualize the features in GBrowse.

Users will find the same navigation and display style of GBrowse. Novice GBrowse users can refer to the GBrowse tutorial available at OpenHelix (http://www.openhelix.com/gbrowse). In addition to the normal GBrowse

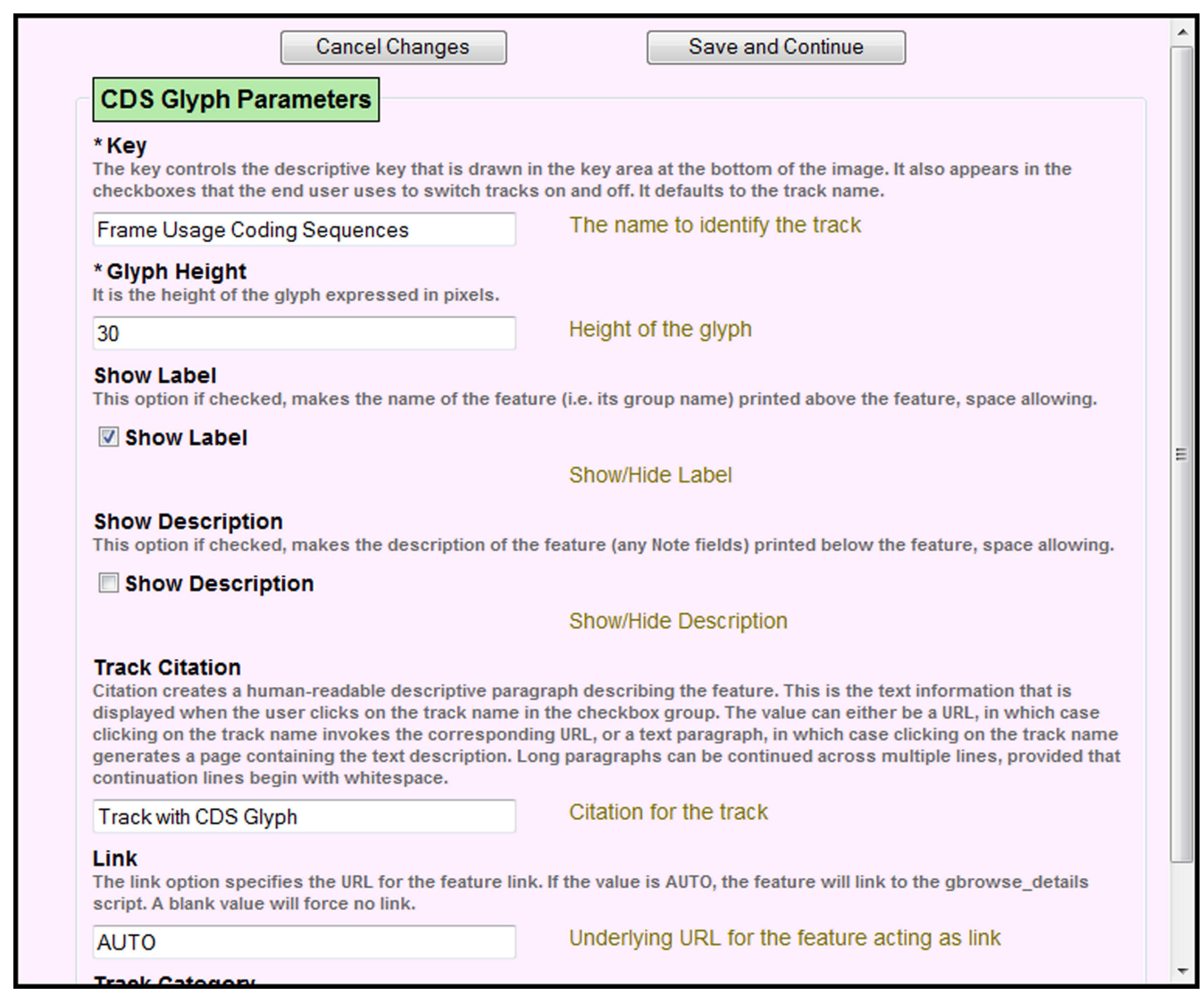

FIGURE 4. The glyph-specific configuration can be edited in the Glyph Parameters Form. 
Select a track from the list on the left and you will see the configuration settings for that feature in the box on the right. Click on Edit Track to edit the configuration settings of the selected track. In case you want to delete the selected track, click Delete Track. Once you are done adding tracks, you can proceed to the actual GBrowse display by clicking on Display in GBrowse. You will be allowed to come back and make further changes if desired.

Configured Tracks *

gene

\section{mRNA}

microarray_oligo
Configuration Settings

Track Configuration for "mRNA"

Glyph: processed transcript

key: mRNA Transcripts

bgcolor: peachpuff

fgcolor: black

height: 10

label: 1

description:

citation: Track with processed_transcript

Glyph

FIGURE 5. All the tracks can be reviewed for further editing or deletion before proceeding to the final GBrowse display.

display, there is a WebGBrowse Control Panel (Fig. 6) displayed at the top of the GBrowse that allows you to return to the Configuration Panel to make further changes or to download the configuration file.

16. To further edit the tracks, click the button "Edit Configuration" in the WebGBrowse Control Panel. This will return you to the Configuration Panel.

17. To save the configuration to a file, click the button "Download Configuration" in the WebGBrowse Control Panel.

This is useful for reusing the same configuration setting as a template for a similar data set, as explained in Step 18.

\section{WebGBrowse Control Panel}

\begin{tabular}{c|c}
\hline Edit Configuration & Click Edit Configuration to return to the track configuration panel \\
\hline Download Configuration & Click Download Configuration to download the configuration file
\end{tabular}

Showing $10 \mathrm{kbp}$ from $\operatorname{ctg} \mathrm{A}$, positions 1 to 10,000

[Bookmark this] [Upload your own data] [Hide banner] [Share these tracks] [Link to Image] [Help]

由 $\underline{\text { Search }}$

由 Overview

曰 $\underline{\text { Details }}$

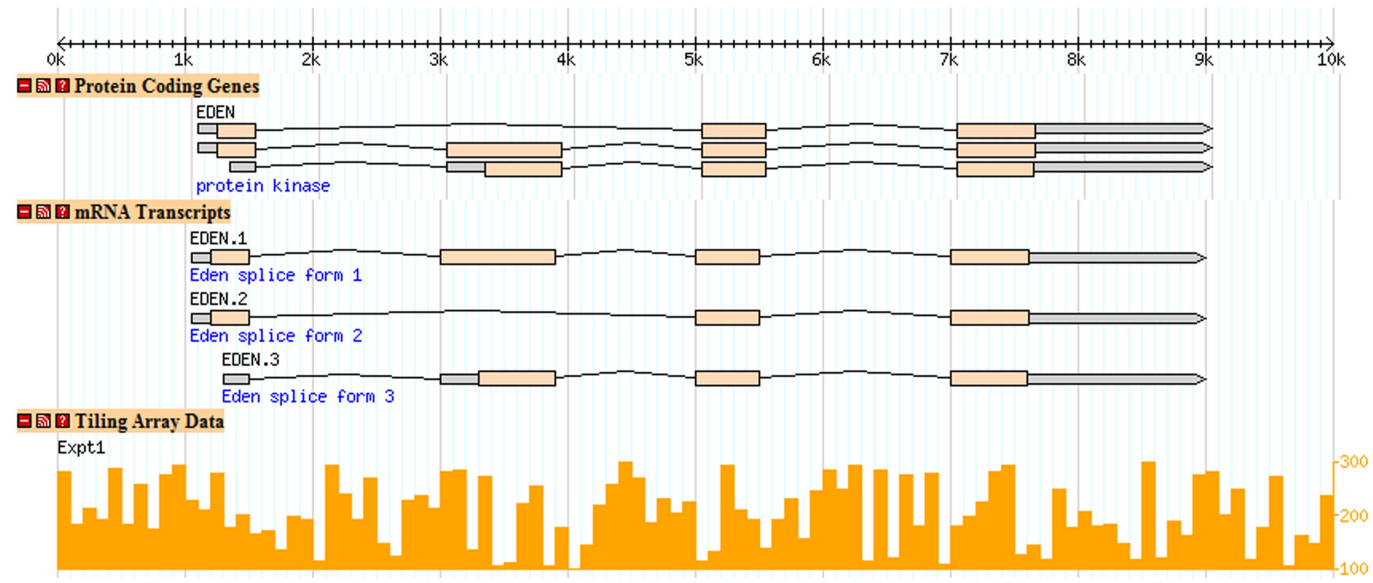

FIGURE 6. Three tracks (i.e., Gene, mRNA, and tiling array hybridization intensity signals) are displayed in this example. Users can use the embedded WebGBrowse Control Panel to edit or download the GBrowse configuration file. 


\section{Using a Configuration Template}

18. To use a pre-existing configuration file as a template to configure another similar data set:

i. After performing Step 3 with the new data set, click the "Browse..." button in the "Configuration File to be used as a template" section of the WebGBrowse Input Form (Fig. 1).

ii. Select a previously downloaded configuration file (see Step 17).

iii. Continue at Step 4.

\section{DISCUSSION}

GBrowse is a sophisticated software package that is typically installed and configured by professional bioinformaticians. WebGBrowse enables biologists to simply upload their own genomic data for visualization within a preinstalled GBrowse system. Through embedded web forms, users can easily configure the display of each genomic feature. For institutions that are interested in installing WebGBrowse locally, instructions are available at http://webgbrowse.cgb.indiana.edu/software.html. WebGBrowse supports the latest GBrowse production version 1.69. Currently, a completely new version of GBrowse, 2.0, is being actively developed for improved user experience. Once GBrowse 2.0 is stable for production use, we will also incorporate it into WebGBrowse. Additionally, to assist biologists who are not comfortable with the GFF3 format specifications, we are developing a web-based GFF3 template generator to facilitate the preparation of the required GFF3 input file.

\section{REFERENCES}

Podicheti R, Gollapudi R, Dong Q. 2009. WebGBrowse-a Web server for GBrowse. Bioinformatics 25: 1550-1551.

Stein LD, Mungall C, Shu S, Caudy M, Mangone M, Day A,
Nickerson E, Stajich JE, Harris TW, Arva A, et al. 2002. The generic genome browser: A building block for a model organism system database. Genome Res 12: 1599-1610. 